\title{
Adaptive and Intelligent Navigation of Autonomous Planetary Rovers - A Survey
}

\author{
Cuebong Wong ${ }^{1}$, Erfu Ya
Strathclyde Space Institute \\ Department of Design, Manufacture and Engineering \\ Management \\ University of Strathclyde, Glasgow \\ G1 1XJ, U.K.
}

\author{
${ }^{2}$ School of Computer Science and Electronic Engineering \\ University of Essex \\ Wivenhoe Park, Colchester \\ CO4 3SQ, U.K.
}

\begin{abstract}
The application of robotics and autonomous systems in space has increased dramatically. The ongoing Mars rover mission involving the Curiosity rover, along with the success of its predecessors, is a key milestone that showcases the existing capabilities of robotic technology. Nevertheless, there has still been a heavy reliance on human tele-operators to drive these systems. Reducing the reliance on human experts for navigational tasks on Mars remains a major challenge due to the harsh and complex nature of the Martian terrains. The development of a truly autonomous rover system with the capability to be effectively navigated in such environments requires intelligent and adaptive methods fitting for a system with limited resources. This paper surveys a representative selection of work applicable to autonomous planetary rover navigation, discussing some ongoing challenges and promising future research directions from the perspectives of the authors.
\end{abstract}

Keywords-planetary rovers, space robotics, machine intelligence, navigation algorithms, autonomous systems

\section{INTRODUCTION}

The rapid development of mobile robotics and other space technology has enabled the exploration of the unknown beyond Earth. Particularly notable achievements in planetary exploration include the successful deployment of several Mars rovers, namely Sojourner, Spirit and Opportunity, and the Curiosity rover [1]. These missions aimed to explore the surface and geology of Mars to discover the history of the planet's water activity, determine the geological processes that shaped the landscape, and assess the habitability of the environment, and much more [2][3]. The success of these missions were considerable feats that demonstrate the benefits that can be derived from the deployment of autonomous wheeled mobile robots to assist and support human activities. However, while the performances of these rovers are nothing short of impressive, there is still large room for improvement.

Developing rover systems capable of exploring the Martian environment fully autonomously is an ongoing challenge. Traditional navigation techniques developed for very particular scenarios on Earth are simply inadequate for dealing with the harsh and challenging environment of Mars. Rather, more generalized, adaptive and intelligent methods are needed to deal with the interactions with unknown landscapes robustly and autonomously. With regard to intelligent methods under consideration in this survey, we refer to a series of soft computing techniques that can be roughly divided into the following categories: neural networks, fuzzy logic, probabilistic methods, evolutionary algorithms (which include genetic algorithm, ant colony optimization and particle swarm optimization) and hybrid methods. In addition to this, recent developments in cognitive robotics are also drawing interest in the field of navigation, where self-learning capabilities provide robots with an ability to adapt to unseen scenarios.

From literature review, we have found some relevant work that begins to offer viable solutions to the problem of navigation on Mars. However, such research efforts are scattered among the widespread research on mobile robotics. The authors in [4] and [5] survey techniques for terrain classification and characterization that demonstrate potential for planetary rover applications. However, to our knowledge, no existing work surveys adaptive and intelligent navigation methods for the broader problem of planetary exploration. To address this gap, this survey paper brings together a representative selection of work in intelligent and adaptive navigation that show potential for use in planetary exploration and gives our perspectives on the promising future directions for the development of more intelligent and autonomous planetary rovers. Thus this paper is organized as follows. Section II provides a scope description of various tasks involved in navigation, while section III introduces the capabilities of existing Mars rovers and the difficulties faced during operation on Mars. In section IV we review a selection of relevant work that is representative of the current state of research. Section V concludes the paper with a discussion of the implications of current methods to the future of planetary rovers.

\section{Planetary Rover Navigation Overview}

The term navigation encapsulates a number of different tasks required of a robotic system. This can be broken up into path 


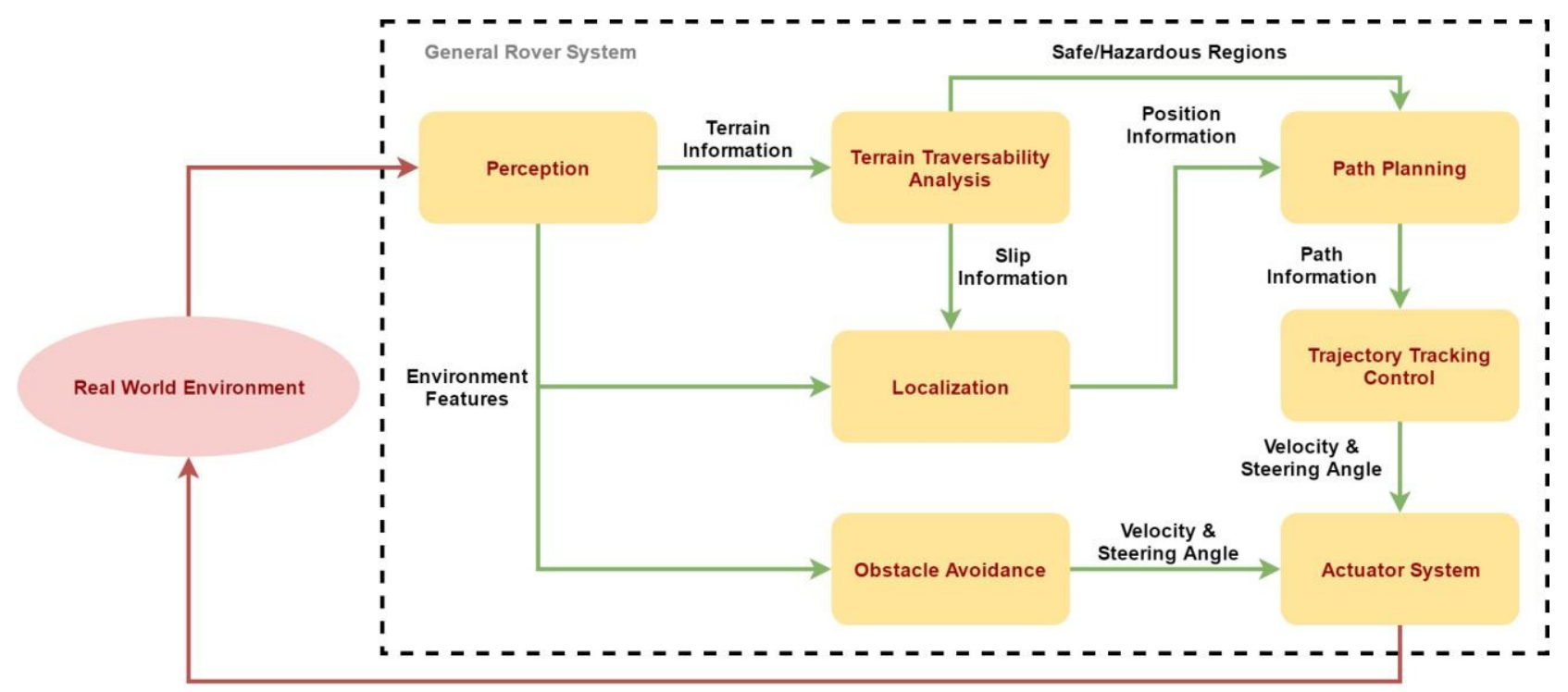

Fig. 1. A general overview of rover navigation system

planning, obstacle avoidance, localization and terrain traversability analysis, etc. Indeed, there are additional tasks performed by robots that relate to navigation, such as trajectory tracking control, dynamic target tracking and object recognition, but we do not cover these aspects in this paper due to page limit. Fig. 1 illustrates the general architecture for the autonomous navigation of a rover system.

Path planning consists of finding a feasible path from a starting pose to a final destination in an optimal manner. Path planning methods can be divided into two classes: global path planning and local path planning. Global path planning seeks to find a high-level path based on some a priori knowledge of the environment, and is effective for generating optimal high level path plans for a rover to execute. However, they are generally inadequate for handling dynamic/unknown environments. On the other hand, local path planning relies on sensory information to ensure global plans are executed accurately and potential collisions are prevented. While it benefits from requiring no priori knowledge of the environment, a local planner is generally insufficient for generating an optimal path to a goal. Many recent approaches to path planning incorporate both global and local path planning for more robust and optimal navigation performance [6].

Obstacle avoidance involves the detection and avoidance of objects/hazards that obstruct the robot as it performs a given task. This requires the use of sensory information from onboard (and sometimes external) sensors, such as vision systems and laser scanners, to detect dangerous features in the robot's vicinity. Many solutions have been proposed for dealing with unknown but static obstacles. However, considerable challenges still exist for cluttered environments or where obstacles are dynamic. This is further complicated by errors and uncertainty associated with sensory devices. Currently, these are commonly addressed by consolidating information from multiple sensors through data fusion.
Localization addresses the problem of identifying the location of the robot within its environment. Approaches to localization include relative methods, which track the robot's motion from a reference point, and absolute methods which employ the use of GPS. However, both of these solutions suffer from errors and inaccuracies which must be compensated for. Hence, an assessment of local landmarks is frequently consolidated with sensors to provide more accurate estimations of the robot's location. This becomes increasingly difficult with harsher environments, where GPS signals are absent and landmarks are not known a priori. An extended activity of localization is Simultaneous Localization and Mapping (SLAM) [7]. This is a process that deals with mapping an unknown environment using a robot while at the same time tracking its own location within it. This map can them be used for navigation. Various sensors may be used to achieve this, such as cameras, laser rangefinders and sonar sensors.

Terrain traversability analysis is a necessary aspect of navigation for applications in unstructured and difficult terrain. In these environments, robots commonly encounter areas that are impassable due to the nature of wheel-terrain interaction. Thus, an important step in navigating through these environments is to classify the type of terrain (e.g. soil/grass) and to characterize their properties to assess the traversability and identify the mechanical properties of the surfaces.

\section{COMMON CHALLENGES}

The most recently deployed Curiosity rover on the surface of Mars has three primary modes of navigation. These are: blind-drive, hazard avoidance, and visual odometry [1]. In blind-drive mode, human operators on Earth use information from local images to identify a safe path for the rover. Commands are sent to drive the rover along this path as far as the operator has deemed safe. The rover tracks the distance travelled through wheel odometry. This mode of operation 
offers the highest traversal speed as the rover does not need to assess the safety of the path. However, the length of the safe path planned by human experts is limited by the amount of terrain information that is assessable from local images. In hazard avoidance mode, the rover autonomously chooses a path to follow such that hazards are avoided. To achieve this, the rover stops at regular intervals to assess the local terrain through images captured with its body-mounted hazard avoidance stereo cameras. The rover then updates its path based on any detected hazards. Similarly for visual odometry navigation [8], the rover periodically stops to check for slippage. Images are acquired from the mast-mounted navigation cameras at each instance and compared with previous images. Similar features are identified and matched to determine the distance travelled. This enables the rover to determine whether it is stuck and can prevent further negative actions from taking place.

While Curiosity and its predecessors have demonstrated success in navigating the Martian terrain, there are still a number of key challenges that exist for planetary rover navigation. The mobile nature of the rovers means that onboard resources are limited. The heavy reliance on imagery for hazard avoidance and visual odometry requires the robot to stop periodically, which is inefficient and restricts the speed of operation [9][10]. Conversely, the need for human intelligence to plan safe paths in blind-drive mode is also restricting due to the limited availability of communications and significant communication delays. Consequently, an area for improvement to the system includes the enhancement of autonomous behavior without the use of computationally expensive approaches. Certainly, by providing the rover with greater goal/path selection capability, human involvement can be significantly reduced in the navigational loop.

Another significant challenge that existing rovers have been faced with is their interactions with hazardous terrains [11]. Mars rovers are required to operate in various unprepared terrains with differing properties. For example, rovers can experience high levels of slippage on particularly steep slopes [12], or dangerous levels of sinkage on terrain with insufficient mechanical strength [10]. Some of these properties are difficult to identify at a distance from visual observations alone. In more extreme cases, it is not until the rover has entered a hazardous region that it was able to classify the terrain. One consequence of this is difficulty in performing trajectory tracking on highslip surfaces [13]. Since the complex wheel-terrain interaction effects are not precisely known, it can be difficult to model such effects accurately. Methods for effective trajectory tracking control where high-accuracy dynamic models of the rover-terrain interaction are not fully known are therefore desirable. Perhaps an even more favorable outcome than this would be to avoid difficult terrain altogether, where possible. Applying terrain classification and characterization information to online planning activities would therefore be necessary, but proves to be a challenge due to computational demand [14].

SLAM, a key activity in any navigational task in unknown environments, is similarly problematic on Mars. Rovers suffer from high slip ratios due to the combined effects of terrain geometry and mechanical properties, resulting in severe dead reckoning errors. When coupled with the absence of GPS, traditional methods for SLAM are simply inadequate. Visual SLAM has drawn attention for general applications in recent years, but it is nevertheless computationally expensive. Furthermore, while visual localization is particularly suited to feature-rich environments, it is inadequate for featureless landscapes. This problem is significantly elevated when simultaneous considerations are given to terrain traversability analysis. As the research community addresses challenges relating to real-time terrain traversability analysis, it is clear to see that there is still a long way to go before a truly effective simultaneous terrain characterization and classification, localization and mapping solution can be realized together with an effective path planning mechanism [15].

While we have not exhaustively described all the problems encountered in Mars exploration missions, it is sufficient to see that adaptive and intelligent approaches to navigation are necessary to overcome hurdles facing robotic exploration. Indeed, currently deployed rovers lack learning capabilities, a feature of growing interest in the field of robotics.

\section{NAVIGATION OF AUtONOMOUS PlanetaRy ROVERS}

\section{A. Path Planning}

In recent years, there has been a rapid growth in the exploration of evolutionary and swarm algorithms for solving the problem of path planning due to their suitability for handling large, complex environments. Traditional methods are computationally expensive when exploring a fine-grid map, and generally require re-planning a path from scratch when a new obstruction is detected. Stochastic methods therefore enable more efficient path planning in such scenarios. Particle swarm optimization (PSO) has been demonstrated successfully for both global and local path planning in [16]. Here in particular, the authors described a method for realizing online path planning, enabling a robot to modify its motion in real time within unpredictable and/or unknown environments. Another notable strength of stochastic methods is their adaptability for hybrid methods. Tusi and Chung [17] combines the artificial bee colony algorithm (ABC) with the rapidlyexploring random tree (RRT) to compensate for the drawbacks of the two individual methods. This led to an approach with faster convergence, improved stability and enhanced ability to avoid entrapment in local minima. Evolutionary and swarm algorithms have also been combined with fuzzy systems and neural networks (these are further discussed below). Algabri [18] presented a comparison of four hybrid methods (namely manual fuzzy, genetic algorithm-based fuzzy, PSO-based fuzzy, and neuro-fuzzy) to assess the performance of these methods relative to each other. The author notes that these methods outperform each other for different aspects and no one method is necessarily superior overall for general applications.

Fuzzy rule sets have been used to address uncertainty in navigation by emulating human reasoning with linguistic terms. 
Fuzzy systems for navigation have evolved considerably since its appearance in early works [19], and recently authors have attempted to improve their robustness through the use of fuzzyhybrid methods. In [20], a dual rule-based fuzzy path planner is presented. Multi-objective stochastic optimization methods are used to scale the parameters in the fuzzification and defuzzification process such that the planned path is time/energy optimal, safe and smooth. Type-2 fuzzy sets [21] have also begun to appear in robot navigation. These systems have improved capability to handle uncertainty and imprecision through the use of upper/lower membership functions to model uncertainty in the degree of membership. Its application in path planning of mobile robots has been demonstrated in [22].

Neural networks have also appeared prominently in mobile robot literature since the late 1990s [23]. Continued research efforts dedicated to this field has led to new classes of neural networks that demonstrate effective performance in navigation compared with other techniques. The work in [24] investigated the performance of a mobile robot using wavelet neural network for navigation. Experimental results showed that this class of neural networks can improve the speed of training and convergence while exhibiting good robustness attributes. Luo et al. [25] addressed the problem of multi-goal motion planning and map building in unknown environments through the use of a Hopfield neural network (HNN). Their approach combines the $\mathrm{D}^{*}$-Lite algorithm and vector field histogram for global and local path planning capabilities together with the HNN to realize multiple navigation objectives. This work demonstrates the feasibility of implementing neural networks to perform multiple tasks concurrently in real-time.

Most recently a number of novel learning-based hybrid algorithms have been trialed for path planning applications. A cognitive learning-based algorithm is presented in [26]. Here an extreme learning machine (ELM), which consists of a single hidden layer neural network that does not require adjustment of weights, is combined with reinforcement Q-learning [27] to enable online self-learning. This proposed algorithm demonstrates good generalization performance and fast learning speeds necessary for real-time applications in diverse/unknown environments. Other reinforcement learning (RL) methods have also been proposed, such as in [28], where a fuzzy logic controller is integrated with heuristic dynamic programming based on a method called Dyna planning. Dyna was proposed by Sutton in 1991 as an artificial intelligence architecture which combines learning, planning and reactive execution [29]. The authors in [28] compared their approach with other RL methods and concluded that this algorithm is capable of generating faster near-optimal paths with high stability, but at the expense of high computational cost. Additionally, a key advantage of the Dyna approach is its scalability into multi-robot systems. However, currently this has only been tested in a maze-like environment and further work is required to extend this to outdoor terrain. Yu et al. [30] addressed path planning in outdoor environments also using ELM. Based on ridge regression (RR) theory, the proposed algorithm determines an optimal path based on distance and takes into consideration dynamic environments. Comparisons with classic support vector machine (SVM) and ELM approaches demonstrate that the RR ELM has better generalization performance, smoothness and speed.

\section{B. Obstacle Avoidance}

Much attention has been given to the use of neural networks to perform obstacle and hazard avoidance. A neural-based autonomous navigation algorithm is presented in [31] for wheeled mobile robots operating in unknown and unstructured environments. By using a single diagonal recurrent neural network (RNN) to optimize the output of a supporting reactive navigation algorithm, the proposed approach enables real-time obstacle avoidance while minimizing the length of the path taken to reach a target, despite having no a priori knowledge of the environment. The network is trained in a two-step supervised off-line learning and online weight-adjustment process, providing it with good generalization performance and guaranteed convergence. Likewise, the authors in [32] presented a hybrid approach to obstacle avoidance, which incorporates a neural network to navigate through cluttered dynamic environments. Using perceived information about the environment across six segments of the robot's field of view, the neural network determines the safest direction to maneuver past obstacles while maintaining a desired traversal direction.

A number of authors have also addressed problems arising from the presence of uncertainties by integrating fuzzy logic methods with neural networks. Noisy sensory information is treated using fuzzy rules before being fed into a neural network in [33]. When compared with a purely neural-based model, the proposed method proved to be superior both in terms of obstacle avoidance success rate and the smoothness of navigation. The use of fuzzy logic for the treatment of uncertainties also benefits from fast computation and easy interpretation. The adaptability of fuzzy logic to dynamic and unstructured environments are further improved upon in [34], where the authors investigated the use of interval type-2 fuzzy sets to handle greater levels of uncertainties. This is combined with a neural network such that the process of fuzzification is handled in the input layer of the neural network. The neural network then deals with the actual obstacle avoidance task. Simulations and experiments verified that the algorithm achieved smooth motion during obstacle avoidance and demonstrated good position stabilization.

Additionally, learning-based fuzzy and neural network approaches have recently been appearing in literature with some proven success. Duguleana and Mogan [35] proposed a Q-learning and neural network algorithm for static and dynamic obstacle avoidance. Motivated by the high computational time of traditional methods, their work enables the effective navigation of a mobile robot with adjustable speeds while avoiding local minima. Furthermore, the hybrid nature of their solution eliminates the drawbacks of Q-learning while retaining the essence of its use in navigation (that is to find optimal paths without previous knowledge of the environment). However, in their study, the authors assumed 
that all dynamic parameters are known at any time instant, which is often not true in real scenarios. The use of Q-learning to provide robots with self-learning capabilities have also appeared in other works. In [36], Q-learning was used with an unsupervised, weightless neural network algorithm for identifying, differentiating and classifying obstacles. With this algorithm, a robot is capable of maneuvering through obstacles by self-learning from experience, much like how a human learns. This proposed method illustrates how learning techniques can be used to overcome problems associated with traditional artificial intelligence methods, namely their thorough training requirements and difficulty with handling new, unseen scenarios. The use of operant learning (OL) (based on animal learning) combined with a probabilistic fuzzy controller is proposed in [37]. The authors address the difficulty of maintaining correctness, consistency and completeness of a fuzzy rule base by employing OL to tune fuzzy set parameters, while uncertainty is handled by probabilistic terms. Indeed, simulation results demonstrate that the system behavior resembles that of animal learning. Consequently, failures and collisions can occur during early stages of navigation but reduces significantly over time.

Initial studies into cognition-inspired approaches have also begun to surface within the research community. The work in [38] introduces the concept of fuzzy cognitive maps (FCM) for a knowledge-based navigation system. By modelling decisionmaking tasks through heuristic knowledge that link navigation concepts and causal relationships together, the system becomes able to adapt to changes in the environment and successfully avoid obstacles. While this method is still in its infancy, initial results provide a glimpse of its future potential for more autonomous navigation applications. Likewise, the feasibility of a cognitive development learning model, implemented through a neural network, for autonomous navigation has been investigated [39]. The author presented a dynamically adjusted neural network, whereby neurons were added or removed such that the biological behavior of living organisms are imitated, to enable the robot to learn from its interaction with an environment. The experimental results, tested on a robotic fish in water, are promising. However, the author reported signs of slight jitter, which implies a sub-optimal trajectory. Certainly, further investigations will be necessary to better understand the performance of this approach, but much like the work in [38], it demonstrates potential for autonomous navigation in complex environments.

\section{Terrain Traversability Analysis}

Truly autonomous rover navigation cannot be realized on environments such as the surface of Mars without first enabling the system to obtain, understand and use terrain information in real time. Wheel sinkage has been identified as a good indicator of the mechanical properties of outdoor terrain [40]. Nevertheless, this method alone does not prevent a rover system from entering hazardous regions entirely. To tackle this open problem, a number of authors have proposed vision-based machine learning techniques to estimate traversable regions in distant terrain. The same authors in [40] developed a classifier framework whereby information from proprioceptive sensors are used to classify the terrain traversed at a particular time instant [41]. The results of this classification, along with visual information of the classified terrain, are then used to train a separate vision-based classifier to enable classification of more distant terrain. The process of classification is mainly performed using SVMs. In a similar fashion, Otsu et al. [42] proposed the use of both vibration-based and visual-based classifiers to operate concurrently. Information obtained from the two sensors is used firstly to classify the terrain using SVMs, and secondly to re-train each other's classifier outputs. This approach enables a greater degree of training from a sparse dataset and has been validated on Martian-like terrain.

In [43], an adaptive feature selection approach to terrain classification, based on the Random Forest method, was presented. Using a self-supervised learning framework to train a visual classifier, key feature information is extracted from images to identify color characteristics, texture information and geometric features associated with the terrain. It was concluded from experimental results that this framework provides good real-time performance. Yet from past experience, it has already been understood that such observations alone are insufficient for robust and accurate assessment of terrain mechanical properties. Fujita and Ichimura addressed this problem in [44], where they devised a framework for the classification of nongeometric terrain properties based on Dynamic Texture analysis. A sequence of images of moving scenes is analyzed to identify soils types and to estimate the rover velocities relative to the terrain, from which the level of slippage can be derived approximately. An additional benefit of this approach is robustness to time-changing features such as lighting conditions, texture and physical configurations of the terrain.

The application of neural networks for terrain traversability analysis has also been promising. Notably, a variety of RNN architectures for visual classification were investigated in [45]. In this work, a visual feature progression technique was utilized to perform mutations, such as scaling and blurring, on feature image patches to generate a sequence of feature vectors. Classification of these feature vectors were then performed using RNN. The authors compared these results with that of Random Forest-based methods and highlighted the superior performance of the RNN approach. Likewise, in [46] the suitability of RNN for vibration-based classification of terrains was studied. The authors combined this with Sequence Boundary Dropout, a regularization technique, to enlarge the training set and improve generalization. The authors discuss the strengths of neural networks when compared with traditional classification techniques, commenting that neural networks classify "in the time domain without any explicit feature computation" [46]. Indeed, the performance of RNNs were verified in experiments, proving successful in a test to identify samples from among 14 terrain classes in real-time.

Most recently, a novel software, Soil Property and Object Classification (SPOC), was developed from deep learning methods [47]. Using visual images of Martian terrain captured both on-ground and in-orbit, a deep convolutional neural 
network (CNN) was employed to classify terrain types and features. While this software required training using data provided by human experts, it was successfully applied to two Mars rover missions: landing site analysis for Mars 2020 Rover mission; and slip prediction modelling using data captured with the Curiosity rover. Currently this software is used independently for off-line terrain traversability analysis. However, its exemplary performance highlights the potential of intelligent techniques for realizing autonomous, real-time terrain classification for planetary rovers.

\section{Localization}

Many authors have addressed the problem of localization in challenging environments. A hybrid approach called R-SLAM, which combines both feature and grid-based methods for the generation of consistent and high resolution maps, is presented in [48]. The work is motivated by a need to overcome both systematic and non-systematic odometry errors (due to uneven surfaces and wheel slip etc.) and addresses the challenge of GPS-denied environments. The hybrid nature of this approach restricts the use of feature-based localization, which reduces computational load. In [49], Havangi investigated the performance of intelligent FastSLAM, which improves upon the original FastSLAM algorithm [50], through the inclusion of soft computing methods. In particular, an evolutionary filter search is applied for best pose estimation; while a fuzzy unscented Kalman filter handles feature location estimation. Experimental results show that this variation of FastSLAM reduces the problem of performance degradation over time as a result of unknown statistical noise. The application of fuzzy logic to localization was further extended in [51], where a neuro-fuzzy system was developed for the purpose of slip compensation. This architecture enables the system to build a model capable of learning slip dynamics, which is then utilized for wheel slip compensation. Through its self-calibrating mechanism enabled by the neural-based implementation, the algorithm demonstrates robustness to terrain variations while improving computational efficiency.

Aside from neuro-fuzzy architectures, (deep) CNNs have also been implemented with the demonstrated success for various visual localization solutions. The effectiveness of CNNs for handling certain aspects of change in an environment was demonstrated in [52], where it is used for long-term localization applications in environments experience seasonal change. Meanwhile, a set of spatial vocabulary was developed from the ontology of space and used to identify features based on CNN implementation in [53]. Both of these works demonstrate potential in the use of CNNs for effective generalized localization performance. However, this is subject to the availability of diverse training data. Nevertheless, there is a good indication that $\mathrm{CNN}$ methods enable localization performance on par with or better than other existing methods.

Bajpai et al. [54] specifically addressed the problem of SLAM for planetary rovers using a biologically-inspired approach. Here a hybrid visual saliency model is developed to enable semantic feature detection using monocular images, which, as a result, reduces the complexity of the system. Three point-based feature techniques were tested within the system architecture to identify the potential effectiveness of the hybrid saliency model. Experimentation on challenging real-world datasets indicated that this solution outperforms visual odometry localization approaches while exhibiting robust and lightweight properties.

\section{DISCUSSION AND FUTURE DIRECTIONS}

It is becoming more widely recognized that as mobile robots venture into more complex and harsh environments, traditional approaches to performing navigational tasks are insufficient. In our survey of emerging solutions to autonomous navigation, it is clear that adaptive, intelligent and more generalized methods play a pivotal role in equipping planetary rovers with the necessary capabilities to interact with the environment in a truly autonomous way.

From surveying the literature, it can be observed that the advancement of machine learning methods have enabled good terrain traversability analysis possibilities that do not rely on human expertise. The development of SPOC is a key milestone that demonstrates the potential of applying deep learning to perform autonomous terrain classification. Though it is still a challenge, with such a feature rover systems would be able to perform accurate and adaptive self-learning through terrain classification. Furthermore, given the consequences of encountering critical situations, the capabilities of SPOC could be further extended to include the characterization of terrain mechanical properties. This will involve the utilization of knowledge gained from the progress achieved by past works exploring the use of proprioceptive sensors in addition to visual information. Looking even further ahead, it will be a necessary task for the rover to incorporate terrain information obtained autonomously and apply it to other activities such as path planning, localization and trajectory tracking control. For the case of path planning, it is naturally desirable to avoid hazards such as regions of high slippage and/or sinkage risk, and rocky surfaces that can damage rover wheels. Similarly, a good estimate of wheel slippage will improve the performance of localization and trajectory tracking capabilities. It has already been noted that the rover is limited in its computational capacity. As a result, achieving these goals come with the additional challenge of using computationally efficient methods to carry out multiple tasks concurrently. The study of learningbased methods and other neural networks techniques [25] will undoubtedly contribute significantly to realizing these needs.

From the perspective of autonomous path planning and reactive navigation, there will be significantly more issues to address as terrain information is included into the loop in realtime. The compromise of planning energy-efficient [55] yet safe (low-risk) paths while avoiding obstacles and minimizing path distance will be an interesting multi-objective optimization problem [20]. This complexity scales further when planning paths such that areas with a higher likelihood of supporting more accurate localization (feature-rich regions, for example) are also considered. The concept of path planning based on 
reducing localization error has been explored by Inoue et al. [15] using only traditional methods. Thus, the minimization of localization error in path planning using more intelligent and adaptive techniques is an open area for investigation. From our survey we have also observed a growing trend in the use of hybrid methods to enhance the navigational competency of rovers. There is little doubt that by integrating compatible solutions together, drawbacks that existed in the use of individual methods may be eliminated. The performance of these architectures is promising as it has been discovered that these systems perform notably well in certain aspects. Yet there are no signs of a globally best approach emerging. Development of hybrid methods will thus involve the consideration of priorities/objectives relating to other aspects such as generalization performance, computational efficiency, robustness to unseen scenarios and uncertainty handling.

Some of the latest research efforts have begun to shed light on the appropriateness of neurobiologically-inspired learningbased methods for autonomous navigation. While current research awareness of this area is still in its infancy, authors investigating these approaches have already demonstrated a glimpse of the feasibility of such solutions. Two particularly notable advantages of learning-based systems are the elimination of long training times and associated training data and their ability to adapt to unseen scenarios. These will indeed be important traits for a system intended to explore the deep unknown. However, these systems too come with their own drawbacks. That is, they are prone to fail (e.g. collide with obstacles) during initial operation. While this is certainly an undesirable effect, the growth of hybrid solutions may enable a separate system to take over during initial operation as the learning algorithm develops an optimal behavior. Ultimately, the current awareness of neurobiologically-inspired methods is still lacking as many approaches have yet to be thoroughly tested or extended to real applications. Nevertheless, techniques such as deep learning and cognitive decision-making models will continue to draw increasing interest among the research community over the next decade.

\section{CONCLUSION}

The upcoming planned deployment of the Mars 2020 rover will likely see another breakthrough of robotic technology for space exploration. The exploration of such a distant environment will be an incredible challenge for years to come. With heavy limitations on resources such as power and communications, there has never been a greater need for truly autonomous exploration systems. Indeed, we do not foresee human operators being completely removed from the loop in exploration missions anytime soon. The wide access to human expertise and unprecedented simulation software on Earth will still be necessary to assist a rover in critical situations. However, the current demand for intensive human labor to perform more standard navigation tasks may certainly be reduced through the development of a truly autonomous navigation system. With this in mind, this paper gives a survey of recent advances in the application of intelligent algorithms and frameworks for planetary rover navigation. The main objective of this paper is to review a selection of work that represents the current state of research in the field. While literature has not been exhaustively surveyed, key achievements and their relevance to addressing common challenges in planetary exploration have been discussed and some future directions on the development of autonomous planetary rovers have been speculated and described based on the state of the art review.

\section{ACKNOWLEDGMENT}

This research is funded by the Engineering and Physical Sciences Research Council (EPSRC) under its Doctoral Training Partnership Programme (DTP 2016-2017 University of Strathclyde, Glasgow, UK).

\section{REFERENCES}

[1] J. P. Grotzinger et al., "Mars Science Laboratory Mission and Science Investigation,” Space Sci. Rev., vol. 170, no. 1-4, pp. 5-56, Sep. 2012.

[2] M. Thomas, J. Clarke, and C. Pain, "Weathering, erosion and landscape processes on Mars identified from recent rover imagery, and possible Earth analogues," Aust. J. Earth Sci., vol. 52, no. 3, pp. 365-378, Jun. 2005.

[3] J. A. Crisp, M. Adler, J. R. Matijevic, S. W. Squyres, R. E. Arvidson, and D. M. Kass, "Mars Exploration Rover mission," J. Geophys. Res. Planets, vol. 108, no. E12, Dec. 2003.

[4] S. Chhaniyara et al., "Terrain trafficability analysis and soil mechanical property identification for planetary rovers: A survey," J. Terramechanics, vol. 49, no. 2, pp. 115-128, 2012.

[5] Y. Gao, C. Spiteri, M.-T. Pham, and S. Al-Milli, "A survey on recent object detection techniques useful for monocular vision-based planetary terrain classification," Rob. Auton. Syst., vol. 62, no. 2, pp. 151-167, 2014.

[6] M. Hank and M. Haddad, "A hybrid approach for autonomous navigation of mobile robots in partially-known environments," Rob. Auton. Syst., vol. 86, pp. 113-127, 2016.

[7] J. J. Leonard and H. F. Durrant-Whyte, "Simultaneous map building and localization for an autonomous mobile robot," in Proceedings IROS '91:IEEE/RSJ International Workshop on Intelligent Robots and Systems 91, pp. 1442-1447.

[8] L. Matthies et al., "Computer Vision on Mars," Int. J. Comput. Vis., vol. 75, no. 1, pp. 67-92, Jul. 2007.

[9] L. Ding et al., "Three-layer intelligence of planetary exploration wheeled mobile robots: Robint, virtint, and humint," Sci. China Technol. Sci., vol. 58, no. 8, pp. 1299-1317, Aug. 2015.

[10] R. E. Arvidson et al., "Mars Science Laboratory Curiosity Rover Megaripple Crossings up to Sol 710 in Gale Crater," J. F. Robot., p. n/an/a, Feb. 2016.

[11] R. E. Arvidson, "Roving on Mars with Opportunity and Curiosity: Terramechanics and Terrain Properties," in Earth and Space 2014, 2015, pp. 165-173.

[12] M. Heverly et al., "Traverse Performance Characterization for the Mars Science Laboratory Rover," J. F. Robot., vol. 30, no. 6, pp. 835-846, Nov. 2013.

[13] D. M. Helmick, Yang Cheng, and S. I. Roumeliotis, "Path following using visual odometry for a Mars rover in high-slip environments," in 2004 IEEE Aerospace Conference Proceedings (IEEE Cat. No.04TH8720), vol. 2, pp. 772-789.

[14] M. Ono, T. J. Fuchs, A. Steffy, M. Maimone, and Jeng Yen, "Risk-aware planetary rover operation: Autonomous terrain classification and path planning," in 2015 IEEE Aerospace Conference, 2015, pp. 1-10.

[15] H. Inoue, M. Ono, S. Tamaki, and S. Adachi, "Active localization for planetary rovers," in 2016 IEEE Aerospace Conference, 2016, pp. 1-7.

[16] R. Solea and D. Cernega, "Online path planner for mobile robots using 
particle swarm optimization," in 2016 20th International Conference on System Theory, Control and Computing (ICSTCC), 2016, pp. 222-227.

[17] Y. Tusi and H.-Y. Chung, "Using ABC and RRT algorithms to improve mobile robot path planning with danger degree," in 2016 Fifth International Conference on Future Communication Technologies (FGCT), 2016, pp. 21-26.

[18] M. Algabri, H. Mathkour, H. Ramdane, and M. Alsulaiman, "Comparative study of soft computing techniques for mobile robot navigation in an unknown environment," Comput. Human Behav., vol. 50, pp. 42-56, 2015.

[19] A. Saffiotti, "The uses of fuzzy logic in autonomous robot navigation," Soft Comput. - A Fusion Found. Methodol. Appl., vol. 1, no. 4, pp. 180197, Dec. 1997.

[20] C. Hong, C. W. Park, and J.-H. Kim, "Evolutionary dual rule-based fuzzy path planner for omnidirectional mobile robot," in 2016 IEEE International Conference on Fuzzy Systems (FUZZ-IEEE), 2016, pp. $767-774$.

[21] O. Castillo, P. Melin, J. Kacprzyk, and W. Pedrycz, "Type-2 Fuzzy Logic: Theory and Applications," in 2007 IEEE International Conference on Granular Computing (GRC 2007), 2007, pp. 145-145.

[22] K. K. Pandey, A. Pandey, A. Chhotray, and D. R. Parhi, "Navigation of Mobile Robot Using Type-2 FLC," Springer, New Delhi, 2016, pp. 137145

[23] S. X. Yang and M. Meng, "An efficient neural network approach to dynamic robot motion planning," Neural Networks, vol. 13, no. 2, pp. 143-148, 2000.

[24] P. kumar Panigrahi and S. Sahoo, "Navigation of autonomous mobile robot using different activation functions of wavelet neural network," in International Conference for Convergence for Technology-2014, 2014, pp. $1-6$.

[25] C. Luo, J. Zhang, G. E. Jan, and H. Mo, "A new hopfield-type neural network approach to multi-goal vehicle navigation in unknown environments," in 2016 IEEE International Conference on Vehicular Electronics and Safety (ICVES), 2016, pp. 1-6.

[26] H. Ren, R. Yin, F. Li, W. Wang, and M. Huo, "Research on Q-ELM algorithm in robot path planning," in 2016 Chinese Control and Decision Conference (CCDC), 2016, pp. 5975-5979.

[27] C. J. C. H. Watkins, C. J. C. H. Watkins, and P. Dayan, "Q-learning," Mach. Learn., pp. 279--292, 1992.

[28] S. Al Dabooni and D. Wunsch, "Heuristic dynamic programming for mobile robot path planning based on Dyna approach," in 2016 International Joint Conference on Neural Networks (IJCNN), 2016, pp. 3723-3730.

[29] R. S. Sutton and R. S., "Dyna, an integrated architecture for learning, planning, and reacting," ACM SIGART Bull., vol. 2, no. 4, pp. 160-163, Jul. 1991.

[30] L. Yu, Z. Long, N. Xi, Y. Jia, and C. Ding, "Local path planning based on Ridge Regression Extreme Learning Machines for an outdoor robot," in 2015 IEEE International Conference on Robotics and Biomimetics (ROBIO), 2015, pp. 745-750.

[31] M. Al-Sagban and R. Dhaouadi, "Neural Based Autonomous Navigation of Wheeled Mobile Robots," J. Autom. Mob. Robot. Intell. Syst., vol. 10, no. 2, pp. 64-72, 2016.

[32] N. Hacene and B. Mendil, "Toward safety navigation in cluttered dynamic environment: A robot neural-based hybrid autonomous navigation and obstacle avoidance with moving target tracking," in 2015 3rd International Conference on Control, Engineering \& Information Technology (CEIT), 2015, pp. 1-6.

[33] R. M. F. Alves and C. R. Lopes, "Obstacle avoidance for mobile robots: A Hybrid Intelligent System based on Fuzzy Logic and Artificial Neural Network," in 2016 IEEE International Conference on Fuzzy Systems (FUZZ-IEEE), 2016, pp. 1038-1043.

[34] C.-J. Kim and D. Chwa, "Obstacle Avoidance Method for Wheeled Mobile Robots Using Interval Type-2 Fuzzy Neural Network," IEEE Trans. Fuzzy Syst., vol. 23, no. 3, pp. 677-687, Jun. 2015.

[35] M. Duguleana and G. Mogan, "Neural networks based reinforcement learning for mobile robots obstacle avoidance," Expert Syst. Appl., vol. 62, pp. 104-115, 2016.

[36] Y. Yusof, H. M. A. H. Mansor, and H. M. D. Baba, "Simulation of mobile robot navigation utilizing reinforcement and unsupervised weightless neural network learning algorithm," in 2015 IEEE Student Conference on Research and Development (SCOReD), 2015, pp. 123128.

[37] Y. Gao, X. Ruan, and B. Li, "A probabilistic fuzzy controller with operant learning for robot navigation," in Proceedings of the 10th World Congress on Intelligent Control and Automation, 2012, pp. 368-373.

[38] M. Mendonca, E. S. da Silva, I. R. Chrun, and L. V. R. Arruda, "Hybrid Dynamic Fuzzy Cognitive Maps and Hierarchical Fuzzy logic controllers for Autonomous Mobile Navigation," in 2016 IEEE International Conference on Fuzzy Systems (FUZZ-IEEE), 2016, pp. 2516-2521.

[39] J. Cai, "The Cognitive Development Learning Model and Its Application in Autonomous Navigation of Mobile Robot," Rev. la Fac. Ing., vol. 31, no. $3,2016$.

[40] C. A. Brooks, K. D. Iagnemma, and S. Dubowsky, "Visual wheel sinkage measurement for planetary rover mobility characterization," Auton. Robots, vol. 21, no. 1, pp. 55-64, Aug. 2006.

[41] C. A. Brooks and K. Iagnemma, "Self-supervised terrain classification for planetary surface exploration rovers," J. F. Robot., vol. 29, no. 3, pp. 445-468, May 2012.

[42] K. Otsu, M. Ono, T. J. Fuchs, I. Baldwin, and T. Kubota, "Autonomous Terrain Classification With Co- and Self-Training Approach," IEEE Robot. Autom. Lett., vol. 1, no. 2, pp. 814-819, Jul. 2016.

[43] H. Zhang, X. Dai, F. Sun, and J. Yuan, "Terrain classification in field environment based on Random Forest for the mobile robot," in 2016 35th Chinese Control Conference (CCC), 2016, pp. 6074-6079.

[44] K. Fujita and N. Ichimura, "Classifying terrain properties for planetary exploration rovers based on a combined distance measure with dynamic texture model," Rob. Auton. Syst., vol. 70, pp. 156-165, 2015.

[45] S. Otte, S. Laible, R. Hanten, and A. Zell, "Robust Visual Terrain Classification with Recurrent Neural Networks," in European Symposium on Artificial Neural Networks, Computational Intelligence and Machine Learning (ESANN), 2015, vol. 23.

[46] S. Otte, C. Weiss, T. Scherer, and A. Zell, "Recurrent Neural Networks for fast and robust vibration-based ground classification on mobile robots," in 2016 IEEE International Conference on Robotics and Automation (ICRA), 2016, pp. 5603-5608.

[47] B. Rothrock, R. Kennedy, C. Cunningham, J. Papon, M. Heverly, and M. Ono, "SPOC: Deep Learning-based Terrain Classification for Mars Rover Missions," in AIAA SPACE 2016, 2016.

[48] M. Balcılar, S. Yavuz, M. F. Amasyalı, E. Uslu, and F. Çakmak, "RSLAM: Resilient localization and mapping in challenging environments," Rob. Auton. Syst., vol. 87, pp. 66-80, 2017.

[49] R. Havangi, "Intelligent FastSLAM: An Intelligent Factorized Solution to Simultaneous Localization and Mapping," Int. J. Humanoid Robot., p. 1650026, Nov. 2016

[50] M. Montemerlo, S. Thrun, D. Koller, and B. Wegbreit, "FastSLAM: A factored solution to the simultaneous localization and mapping problem," in Proceedings of the National Conference on Artificial Intelligence, 2002.

[51] S. Rakesh kumar, K. Ramkumar, S. Srinivasan, V. Emilia Balas, and F Shi, "Self-Calibration of Dead Reckoning Sensor for Skid-Steer Mobile Robot Localization Using Neuro-Fuzzy Systems," Springer International Publishing, 2017, pp. 541-551.

[52] R. Arroyo, P. F. Alcantarilla, L. M. Bergasa, and E. Romera, "Fusion and binarization of CNN features for robust topological localization across seasons," in 2016 IEEE/RSJ International Conference on Intelligent Robots and Systems (IROS), 2016, pp. 4656-4663.

[53] R. Goeddel and E. Olson, "Learning semantic place labels from occupancy grids using CNNs," in 2016 IEEE/RSJ International Conference on Intelligent Robots and Systems (IROS), 2016, pp. 39994004

[54] A. Bajpai, G. Burroughes, A. Shaukat, and Y. Gao, "Planetary Monocular Simultaneous Localization and Mapping," J. F. Robot., vol. 33, no. 2, pp. 229-242, Mar. 2016

[55] S. Martin and P. Corke, "Long Term Optimisation of a Mobile Robot with Proprioceptive Perception - Semantic Scholar," in Australasian Conference on Robotics and Automation 2015, 2015. 